\title{
EL PROBLEMA DE LA “ILEGITIMIDAD SEXUAL” POPULAR EN CHILE. 1927-1937
}

\author{
Javiera Poblete Vargas ${ }^{1}$
}

\section{Resumen/Abstract}

En Chile, la conformación del Estado Asistencial Sanitario, en 1925, promovió el desarrollo de artículos científicos, donde los médicos y las "visitadoras sociales", expusieron su preocupación por uno de los problemas sociales - que a su juicio- afectaba la vida y la salud del pueblo, y que ponía en riesgo la estabilidad política y económica del país: la ilegitimidad sexual popular. En este trabajo historiográfico, con metodología documental y archivística, analizaremos el discurso médico y asistencial de la década de 1927 a 1937 sobre la sexualidad popular, con el fin de determinar por qué las conductas sexuales del pueblo, fueron definidas como un problema político y económico.

Palabras clave: género, sexualidad, familia, saber, poder

THE PROBLEM OF “SEXUAL ILLEGALITY” POPULAR IN CHILE. 1927-1937

In Chile, the conformation of the sanitary state in 1925 promoted the development of papers, where doctors and "social visitors" expressed their concern for one of the social problems that, in their opinion, affected the life and health of people, and risk the political and economic stability of the country: the people's sexual illegitimacy. In this historiographic work, with documentary and archival methodology, we will analyze the medical and care discourse of the 1927-1937 decade on popular sexuality, to determine why people's sexual behaviors were defined as a political and economic problem.

Keywords: Gender, Sexuality, Family, Knowledge, Power

\footnotetext{
${ }^{1}$ Chilena, Universidad Autónoma de Madrid. E-mail: javiera.pobletevargas@gmail.com
} 


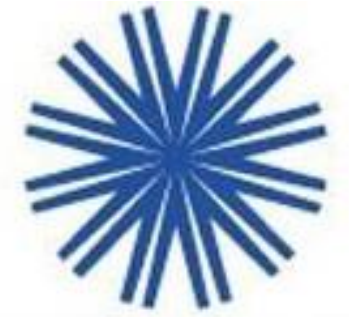

\section{Introducción}

En las primeras décadas del siglo XX chileno, el cuerpo y la sexualidad del pueblo, se convirtieron en un blanco de intervenciones médicas y asistenciales que buscaron fortalecer un nuevo proyecto político. En ese escenario, las malas condiciones de vida del pueblo, sumadas a la propagación de enfermedades y a la masificación del alcoholismo, aumentaban año a año los índices de mortalidad general e infantil; pero además de ello, aumentaban la fuerza del movimiento obrero organizado que buscaba recuperar la vida y la salud del pueblo a través de la gestación de un nuevo orden social que fuese capaz de desestructurar el Estado Oligárquico que durante años había promovido la explotación económica, la exclusión social y política del pueblo. Esta situación se convirtió en un llamado de alerta para la clase dirigente y los sectores medios que temían que se llevara a cabo un proceso revolucionario que afectara las bases de la economía capitalista y del Estado.

Es así, como hacia 1925, se funda el Estado Asistencial Sanitario y con él un proyecto político integracionista, que buscó reestablecer los lazos rotos del pueblo con la clase dominante e integrar al pueblo al Estado; a través de la recuperación de su vida y su salud (Illanes 1993). Para ello, nuevos/as actores sociales fueron los/as responsables de diseñar estrategias biomédicas y asistenciales para dar solución a los problemas médicos y sociales del pueblo. Un nuevo pacto político que buscó disciplinar a la aristocracia, a los empresarios capitalistas, al pueblo y a las propias instituciones públicas, con el fin de subsanar el daño causado por el capitalismo; sin tocar, por supuesto, las condiciones productivas de la misma economía capitalista.

Como resultado de ello, tanto médicos como "visitadoras sociales" asumieron la tarea de reconstruir la sociedad. Su preocupación por la vida y la salud del pueblo y su capacidad científica para comprender el origen de sus males, les permitió incidir en la creación de políticas de intervención y reordenamiento de las fuerzas sociales; y con ello levantar un nuevo desafío biocientífico que, según M. Angélica Illanes, fue definido como:

Una misión salvadora de la civilización occidental, planteado y practicado como una "guerra por otros medios": guerra conducida por oficiales "de delantal blanco" 
y cuyas huestes (femeninas) avanzaban por el campo de batalla a pacificar las iras provocadas por la miseria y la represión. (2006: 26)

De esta forma, los cuerpos dañados del pueblo se convirtieron en blancos de intervención, cambio y factores de política nacional. La intelectualidad médica y asistencial asumió una tarea política-científica que buscó, desde la legalidad y el saber científico, construir las bases de su propio proyecto político.

Lo significativo de la década de 1927 a 1937, es que fue un periodo donde se buscó dar solidez a un proyecto político y social en medio de una profunda crisis económica. En 1929, Chile ocupó el primer lugar del ranking mundial de los países afectados por la caída de la bolsa de valores de Wall Street, y por consecuencia, los efectos en la población más pobre se dejaron sentir durante toda la década del ’30 (Salazar y Pinto 2002). Como consecuencia de ello, los problemas médico-sociales se masificaron y la crisis política se agudizó. Es por eso que los médicos y las visitadoras sociales se esforzaron por comprender al pueblo, por explicar científicamente, los factores que incidían directamente en su realidad.

Así pues, a lo largo de la década escribieron una serie de artículos en diversas revistas de circulación científica provenientes de organismos del Estado y de las escuelas de Servicio Social, donde expusieron sus diagnósticos sobre las causas de los problemas de la época y que daban forma a la realidad médicosocial chilena (Allende 1939), y que tanto desestabilizaban el país. Ahora bien, lo relevante de este ejercicio intelectual es que ambos grupos de profesionales coincidieron en sus artículos, que la falta de control de los impulsos sexuales y la ilegitimidad de las relaciones sexuales de los hombres y mujeres del pueblo, contribuía directamente en la proliferación de la mortalidad, la enfermedad y la miseria. Por tanto, concluyeron que no solo una mejora salarial o económica transformaría sus condiciones de vida, sino que resultaba necesario educarles y guiar sus impulsos sexuales hacia la conformación de un hogar familiar. Solo de este modo sería posible encauzarlos hacia el bienestar moral/social que permitiría recuperar su vida y su salud, pero que también aseguraría el progreso de la nación y apaciguaría el descontento social. De este modo, la sexualidad popular aparece en el discurso científico como uno de los espacios fundamentales para resolver la crisis e incorporar a los sujetos populares al Estado. El objetivo sería desde entonces, difundir en el pueblo el matrimonio y la familia heterosexual monoparental como un modelo ideal de sexualidad. 
Dicho esto entonces, sobre la base de los estudios de género y con algunos aportes de la filosofía política y el análisis crítico del discurso, este trabajo historiográfico se ha propuesto analizar el discurso médico y asistencial sobre la "ilegitimidad sexual popular" que quedó registrado en cuatro revistas médico-sociales entre 1927 y 1937: Servicio Social², Beneficencia ${ }^{3}$, Asistencia Social ${ }^{4}$ y Acción Social ${ }^{5}$; para determinar por qué las conductas sexuales del pueblo, fueron definidas por los médicos y "visitadoras sociales" como un problema político y económico. Por medio de esta acción, intentaremos demostrar que existió un momento en la historia de Chile, en que la sexualidad del pueblo fue tomada como objeto de estudio y explicada como un problema político y económico en la medida en que no se ajustaba a los principios ideológicos -morales y sexuales- de los médicos y visitadoras sociales.

De este modo, intentaremos mostrar que la producción de diagnósticos científicos para la comprensión de las conductas sexuales del pueblo estuvo atravesada por categorías de género y modelos ejemplares de comportamiento sexual, propias del mundo de los/as investigadores, más que de los hombres y mujeres que fueron estudiados. Nos interesará demostrar que categorías como maternidad, paternidad y familia, operaron ideológicamente en el discurso científico para favorecen la producción de un orden simbólico e instaurar en el imaginario colectivo, modelos ejemplares de conducta sexual y de socialización de la libido, que fueron funcionales al proyecto político y económico de los médicos y las visitadoras sociales.

Para ello, desarrollaremos dos apartados que nos permitirán reconocer: en primer lugar, el discurso médico y asistencial sobre la "ilegitimidad sexual popular" presente en los artículos analizados; y en segundo lugar, la ideología moral sexual de los médicos y visitadoras que -desde la perspectiva de nuestro análisis- les permitió explicar la sexualidad popular como un problema político y económico que ponía en riesgo el desarrollo del país.

\section{El problema de la "ilegitimidad sexual" popular}

\footnotetext{
${ }^{2}$ Revista perteneciente al Órgano de la Escuela de Servicio Social de la Junta de Beneficencia de Santiago. Santiago de Chile. Años de circulación: 1927- 1969.

${ }^{3}$ Revista perteneciente al Órgano de la Dirección General de Beneficencia y Asistencia Social. República de Chile; Ministerio de Bienestar Social. Años de circulación: 1929-1932.

${ }^{4}$ Nueva serie de la revista de Beneficencia Pública. Órgano de la Asociación chilena de Asistencia Social. Santiago de Chile. Años de circulación: 1932-1944.

${ }^{5}$ Revista perteneciente a la Caja de Seguro Obligatorio (Ley N 4054) y publicada en Chile entre 1930 y 1948.
} 
En las revistas analizadas, los médicos y las visitadoras sociales explicaron que todas las relaciones sexuales entre hombres y mujeres que se experimentaban fuera de los marcos legales del matrimonio civil, eran ilegítimas. Para ellos/as, estas conductas sexuales debilitaban la vida y la salud del pueblo y exponían a miles de personas a los flagelos de las enfermedades venéreas, la propagación de los vicios, la delincuencia, la mortalidad infantil, los abortos, la prostitución y una serie de problemas médico-sociales que terminaban por agudizar la crisis y el descontento social.

Como consecuencia de ello, la sexualidad del pueblo se convirtió en materia de investigaciones científicas sostenidas en los principios teóricos de la sociología de la época. En sus artículos, los y las profesionales de la medicina y del servicio social, afirmaron que no bastaba con comprender los factores económicos que debilitaban al pueblo y que promovían el descontento social, sino que además resultaba necesario comprender las trabas morales que impedían que el pueblo alcanzara un bienestar colectivo superior.

Según los médicos y las visitadoras sociales, su contacto directo con el pueblo en los conventillos y en hospitales o consultorios, les había permitido reconocer que al interior de la vivienda popular se masificaba en los cuerpos de hombres y mujeres una crisis moral/sexual sin proporciones que los/as llevaba a establecer relaciones sexuales espontáneas, libres e ilegítimas, sin reparar en las graves repercusiones de estos actos al interior del cuerpo social. Para ellos/as, lamentablemente en los conventillos:

Padres, hijos, parientes y allegados duermen en un espacio muy restringido, disponiendo de una, a veces dos camas o jergones, lo que explica el número crecido de casos de incesto, violación de menores que existen en este medio. Si de la habitación pasamos a considerar a la familia, constataremos que el $80 \%$ más o menos de los niños llevados a la justicia pertenecen a familias incompletas o desunidas. Si el padre ha abandonado el hogar, suele ocurrir que la madre viva en concubinato sucesivamente con varios hombres, y no es raro el caso en que uno de ellos llega a ser seductor de la hija de su compañera. (De Bray 1933: 150).

A su parecer la falta de condiciones materiales mínima para su desarrollo y las dificultades económicas propias del escenario de crisis, afectaban la vida del pueblo. Pero el absoluto desconocimiento de sus deberes y sus responsabilidades sociales/sexuales, impedía la construcción de la familia legalizada por el Estado y, por consiguiente, agudizaba la crisis social y económica. 
Si observamos la tabla 1, podemos percibir que las tasas de nupcialidad marcaron un retroceso en los primeros años de la década de 1930, justo cuando la gran crisis del capitalismo mundial tuvo grandes repercusiones en la economía nacional y empeoró las condiciones de vida del pueblo. Para los/as profesionales, estos datos demostraban que los factores económicos dificultaban la conformación familiar en el mundo popular, pero que solo una moral sexual fuerte podría modificar las cifras de nupcialidad. Por eso, aseguraron que resultaba necesario instaurar en el pueblo, una moral sexual que dirigiera sus impulsos hacia la conformación de una familia, ya que solo así sería posible revertir los efectos materiales y morales de la crisis al interior del mundo popular.

Tabla 1. Nupcialidad. Promedio anual por mil habitantes.

\begin{tabular}{ll}
\hline AÑOS & NUPCIALIDAD \\
\hline $1926-30$ & 9,3 \\
1928 & 11,6 \\
1929 & 10,1 \\
1930 & 9,2 \\
1931 & 6,7 \\
1932 & 6,6 \\
1933 & 6,7 \\
1934 & 7 \\
1935 & 7,3 \\
1936 & 7,5 \\
\hline \hline 1937 & 8,3 \\
\hline
\end{tabular}

De esta forma, coincidieron que tanto los factores económicos como morales propiciaban la falta de responsabilidad sexual entre hombres y mujeres del pueblo; pero también lo hacía la falta de conocimiento y la poca importancia que éstos le atribuían al matrimonio y a la familia como la base de toda sociedad. Según el diagnóstico de la Srta. Mac Auliffe, el pueblo tenía una:

a) Carencia absoluta de hábitos higiénicos.

b) Falta de concepto acerca del matrimonio y del hogar como instituciones de trascendencia social. 
c) Absoluta falta de responsabilidad del hombre frente al hogar que él forma.

d) Inconsciencia de la mujer para formar una unión sin exigir que para ello se le presente siquiera una pieza con los útiles más indispensables para comenzar su nueva vida. (1932: 294)

Por su parte, la visitadora social Mme. M. Mulle (1930), afirmó que el matrimonio era una institución de trascendencia social, ya que en la relación entre hombres y mujeres se constituía la familia como base de la sociedad que cargaba en su interior los principios de educación social y moral de los hijos que nacieran de esa unión, de los futuros ciudadanos y trabajadores; pero a su vez garantizaba que hombres y mujeres, por medio de un contrato matrimonial, cumplieran con sus obligaciones sociales que implicaban entre otras cosas, el reconocimiento legal de los hijos por parte del padre y el cuidado materno de los/as niños/as desde su infancia. El problema, era que lamentablemente, tal como planteaba la señorita Mac Auliffe (1932), el pueblo desconocía que el instinto sexual y la familia eran la base de la acción moral y social.

Según los y las profesionales, al interior del mundo popular existía una falta de armonía entre el instinto sexual y la forma en que éste se debe ejercer, pues en su ignorancia, no comprendían que las relaciones sexuales requieren de un grado de responsabilidad social. Para el Dr. Eduardo Rioseco, por ejemplo, el instinto sexual se había convertido sólo en un acto voluptuoso que tenía graves consecuencias físicas y morales al interior del cuerpo social. Ante esto advierte:

La vida social moderna, complicada y difícil, ha traído esta desarmonía entre el instinto sexual y la forma en que se debe ejercer. El acto sexual ha perdido en el concepto de la juventud su finalidad principal, que es la procreación y se ha trocado, sólo en un acto voluptuoso. Las trabas del matrimonio han traído el libertinaje sexual y la prostitución con sus graves consecuencias físicas y morales. (1934: 324).

Por ese motivo, concluyeron en sus artículos, que la inexistencia de un contrato social/sexual matrimonial entre hombres y mujeres, contribuía a que ambos se unieran solo por el hecho de la inclinación natural o del instinto sexual, sin tener un control legal de la vida conyugal que determinara la responsabilidad que cada una de las partes debía tener en el acto de unirse sexualmente con otro. Ante esto, la Srta. Mac Auliffe, diagnostica que:

En suma: el hombre y la mujer se unen por el solo hecho de la inclinación natural del uno por el otro, lo cual hace que en la vida conyugal también sólo se rijan ambos 
por tendencia natural, sin control alguno, sin detenerse ante las consecuencias que este sistema trae consigo (...). (1932: 296).

Como resultado de ello, afirmaron que una vida sexual normal debía resumirse a los hombres y mujeres del pueblo desde la infancia en las palabras: “Unión, Amor, Maternidad, Paternidad, Familia” (Rioseco, 1934: 323). A su juicio, éstas eran la clave para conformar una sociedad en la que hombres y mujeres buscaran satisfacer sus instintos sexuales al interior de la familia y del matrimonio.

Para el médico Eduardo Rioseco, el acto sexual -necesariamente- requería de un control e imponía una responsabilidad a cada persona, porque no era patrimonio del individuo, sino que también pertenecía a la especie misma. Por ende, el ser humano no podía disponerlo a su antojo (Rioseco 1934: 322). Como consecuencia de ello fue común que ambos grupos de profesionales coincidieran en que el acto sexual necesitaba regularse, educarse, controlarse por medio de la asignación de unos deberes sociales y sexuales para hombres y mujeres al interior de la familia, para alcanzar el bienestar de toda la colectividad. El problema era que -como vimos- para los médicos y visitadoras, tales deberes y la importancia de la familia, eran absolutamente desconocidos dentro del pueblo.

En sus artículos, hemos podido observar que el hombre del pueblo fue definido sexualmente como irresponsable. Su incapacidad de constituir familia radicaba principalmente en su condición laboral de jornalero (sin un trabajo estable en un lugar fijo), que le llevaba a cambiar permanentemente de barrio y de ciudad. El problema de ello - afirmaron- era que para él:

Amar a una mujer es cuestión de hacerla suya, no se preocupa de las leyes, mucho menos de la sociedad... los suyos han hecho ya otro tanto... y, así se habitúa el hombre a hacer esas uniones libres que hacen que Chile tenga ilegitimidad en el $37 \%$ de la población. Él cambia de barrio, cambia de pueblo en busca de la faena del momento y, desgraciadamente, también cambia frecuentemente de mujer. (Mac Auliffe 1932: 296).

Esta realidad del hombre popular, incidía - a juicio de los médicos y las visitadoras sociales- en que éstos no asumieran su responsabilidad frente a la paternidad y a la familia. Por lo general y como resultado de su vínculo sexual espontáneo con una mujer, nacía un hijo que jamás reconocían como propio. Además, guiados por sus impulsos sexuales, su libertad y falta de responsabilidad, acudían a los prostíbulos y en ellos adquirían infecciones o enfermedades venéreas que luego transmitían a otras mujeres del pueblo por medio de sus uniones libres. De hecho, las estadísticas demostraban que "un gran número de enfermos de 
esta categoría [enfermedades venéreas] había adquirido tal enfermedad en los prostíbulos, en sus contactos sexuales, o en la promiscuidad del hogar obrero donde estas enfermedades se mancomunaban”. (Canturial y Rodríguez 1936:. 23).

Junto con ello, la conducta sexual de las mujeres también fue cuestionada. A juicio de los/as profesionales, ellas tampoco reparaban en su responsabilidad sexual. Guiadas por sus impulsos sexuales y por las leyes naturales del amor, desconocían su deber maternal al interior de la sociedad. En palabras del Dr. Eduardo Rioseco, desconocían que las mujeres "son sólo los medios de que se vale la naturaleza para que ellas lleguen a ejercer la noble y bella función maternal, dentro del matrimonio” (1934: 324).

Así pues, los médicos y las visitadoras sociales coincidieron en que las uniones ilegítimas tenían vastas y fatales consecuencias en el vínculo que unía naturalmente a la madre con el niño que nacía. Según ellos/as este factor incrementaba las tasas de mortalidad infantil de la época y explicaron que la falta de responsabilidad del hombre con su descendencia, provocaba que las mujeres del pueblo asumieran solas la crianza, mientras que él se mantenía en completo misterio. El problema era que las malas condiciones de vida, la falta de educación y la pobreza en la cual se encontraban inmersas, las obligaba a abortar o abandonar a sus hijos en los conventillos, en hogares de expósitos y en hospitales, sin reparar en el sufrimiento y en el daño moral que les causaban o peor aún, las obligaba a suprimir la vida del hijo que engendraban poniendo en peligro su propia vida:

Por lo general, el niño ilegítimo no cuenta sino con su madre; para ésta, el hijo representa siempre una carga y casi siempre es la desgracia, dentro de nuestra actual legislación. La madre soltera es una mujer repudiada en su casa y en el medio social. Debe trabajar para subsistir y para que subsista su hijo y, por lo tanto, debe destetarlo y abandonarlo a manos mercenarias que, por lo general, no tienen preparación ni interés especial en cuidarlo. Destete prematuro, mala alimentación, abandono. Pero no es esto todo, lo que sería si siempre naciera este fruto de un amor fuera de la legalidad, pero es que en la mayor parte de los casos no nace y tenemos la aparición de un tremendo problema social, cual es el del aborto criminal, causa de gran mortalidad entre las mujeres y de gran disminución de la natalidad. Varios problemas que resolver se perfilan. (Recchione 1935: 578).

El niño y la niña, inocentes de todo mal, quedaban expuestos a sufrir las consecuencias del amor espontáneo de sus padres que nunca repararon en la necesidad de conformar un hogar para darle un sustento social, económico y moral a esta nueva criatura. Así pues, para los médicos y las visitadoras 
sociales el problema de la nefasta fisonomía de los hogares del pueblo, era que ésta contribuía en una sobrecarga del gasto social. Pues, lamentablemente, la sociedad en su conjunto debía asumir el cuidado de los/as niños/as que habían nacido fuera del matrimonio y que vivían abandonados en las calles o en las casas de expósitos. Por eso coincidieron en que era necesario legalizar las uniones amorosas, para depositar gran parte de la responsabilidad social en los padres naturales:

Poco a poco dos consideraciones se han impuesto: una es que el niño nacido fuera del matrimonio es inocente de todo mal y tan merecedor como los otros de gozar de los beneficios de que disfrutan. La otra es la de que; pagando dichos niños con la enfermedad y la muerte las consecuencias de la falta de un hogar, la sociedad se ve gravada de una enorme sobrecarga de acción social por causa de estos niños; sobrecarga que sería en gran parte aminorada si la responsabilidad por el cuidado de los niños nacidos fuera del matrimonio recayese sobre sus padres naturales. (Valdés 1928: 193).

Otro problema de los hombres y las mujeres del pueblo, era que su comportamiento sexual desenfrenado muchas veces era el resultado del abuso de alcohol y estupefacientes que les impedían evaluar los efectos de una relación sexual ilegítima. Así pues, sostenidos en los principios de la teoría eugenésica, la intelectualidad médica y asistencial afirmó que bastaba una intoxicación pasajera de los hombres y mujeres del pueblo, para que de su unión sexual nacieran enfermos, alcohólicos, prostitutas, anormales, etc. (De Bray 1933: 148). Por eso afirmaron que la inmoralidad y el vicio de los padres, les hacían perder la noción de los deberes y también destruían la vida del hogar con sus fatales consecuencias (Gajardo, 1929: 203).

Lo que nos parece significativo es que, desde la perspectiva de los médicos y las visitadoras sociales, la legalización de las uniones sexuales del pueblo mejoraba completamente sus condiciones de vida y por lo mismo, posibilitaba una transformación social. De hecho, Raquel Carrasco, una iniciada en el Servicio Social; manifestó que, en uno de sus casos sociales, la constitución y legalización de la familia fue el elemento clave para la resolución de los problemas que afectaban a los individuos:

Esta familia no estaba constituida, el padre era un borracho, la madre no tenía nociones de sus deberes maternales, la casa era un antro de miseria; ahora está legalizada la unión, los niños van al colegio, la guagua duerme sola en su cunita, todo está aseado, se alimentan económica y racionalmente, el padre trabaja y ayuda a su familia, etc. (Carrasco, 1928: 12). 
Así pues, afirmaron que mientras no hubiese uniones legales, la miseria del pueblo se reproduciría en la ilegitimidad de los amores populares inmorales y libertinos, guiados por el impulso sexual y no por la razón científica que reconocía a éste, como una de las causas de los males sociales en época de crisis.

Como resultado de todos estos factores, la sexualidad del pueblo fue tomada como objeto de análisis y como blanco de intervención; puesto que a través de los diagnósticos elaborados por médicos y visitadoras se estableció que solo una transformación de sus conductas tendría un impacto positivo en la economía y en la consagración de su proyecto político. Por este motivo, amparados en la ciencia, los médicos y las visitadoras sociales asumieron la tarea de producir un saber sobre las conductas sexuales ideales que debían guiar el comportamiento de los hombres y mujeres. Un saber que - desde nuestra perspectiva- fue elaborado a partir de una ideología moral/sexual que buscó legitimar la familia legal, monoparental y heterosexual, para alcanzar el desarrollo de toda la colectividad. Ya que como expuso Samuel Gajardo (1929):

la familia es la célula orgánica de la civilización y todo ataque a esta institución sagrada tiende a disminuir la fuerza de resistencia de la sociedad y facilita, por tanto, la tarea perversa de sus destructores. (1929: 231).

\section{La familia como ideología política-sexual}

El reconocimiento oficial de la satisfacción sexual, independiente de la procreación, sería el golpe que diera al traste con toda la ideología oficial y eclesiástica en materia sexual.

W. Reich ${ }^{6}$

Pues bien, lo que ahora nos convoca es determinar la ideología moral sexual que le permitió a los médicos y visitadoras sociales, explicar la sexualidad popular como un problema político y económico. Y ante esto podemos decir que si bien los/as profesionales no escribieron ningún artículo que abordara directamente nuestro objetivo, podemos realizar un análisis crítico del discurso sobre el tratamiento científico/teórico que éstos/as hicieron de la "ilegitimidad", a partir de ciertas problemáticas histórico-teóricas que nos permitan elaborar algunas conclusiones frente a este problema.

\footnotetext{
${ }^{6}$ Reich, W. 1985. La Revolución sexual. Para una estructura de carácter autónoma del hombre. Editorial Artemisa, México: 63.
} 
Preliminarmente el análisis de las fuentes históricas, nos ha permitido visualizar que existió una desarmonía entre el discurso científico y la forma en cómo los sujetos populares experimentaron su sexualidad. Esta situación demuestra que la producción de conocimiento científico sobre la sexualidad popular, legitimado por el Estado y las instituciones que produjeron las revistas que analizamos; construyó una realidad - supuestamente objetiva- a partir de una ideología moral sexual que definió las conductas sexuales del pueblo como un problema político y económico, sin contemplar ni validar la comprensión del cuerpo y la sexualidad del mundo popular. Es decir, a través de los artículos, pusieron en circulación un discurso sobre la sexualidad humana que clasificó de inmorales las pasiones sexuales populares por ser antagónicas a su propia ideología moral/sexual. Por eso afirmaron que eran un mal que socavaba las bases del progreso nacional.

A razón de esto, partimos de la base que el discurso científico sobre la sexualidad popular emergió de los/as investigadores/as, de su ideología y comprensión de la familia y del matrimonio como instituciones de trascendencia social. No de los sujetos populares que fueron objetivados en la investigación científica de la época. Es por ello que ante la mirada de los/as expertos/as: "se estaba frente al mal que corroía las entrañas de la patria", y su "tratamiento social constituía un deber de alta política." (Illanes 2006: 416).

Con esto nos referimos a que por medio de la técnica de investigación científica sujeta a hipótesis comprobables en su relación “objetiva” con los hombres y las mujeres del pueblo, a datos estadísticos, problemas medibles, cuantificables y a partir del estudio de los denominados "casos sociales"; los médicos y las "visitadoras sociales" buscaron demostrar la validez de su ideología y por medio de esta acción, establecer una verdad que fuese capaz de determinar lo que era correcto y lo que no, lo normal o anormal, moral o inmoral, etc. De esto podemos extraer que la ideología moral/sexual de los/as profesionales se localizó directamente en el saber científico y social sobre la sexualidad popular y en función de esta acción, definieron su problema de investigación, sus objetos de estudio, etc. y presentaron resultados. Fabricaron un saber que de antemano ya fue incitado por la ideología de los/as investigadores (Foucault, 2009). En este sentido, la ideología política/sexual de los médicos y las visitadoras sociales les llevó a clasificar como ilegítimo el comportamiento sexual del pueblo y validar a la familia como la célula constitutiva de la sociedad, que - desde su perspectiva- les permitiría dar solución a los males de la época. 
Ahora bien, en la medida que la familia monoparental heterosexual fue el marco ideológico moral/sexual desde donde los médicos y "visitadoras" observaron al pueblo, podemos visibilizar por qué la "ilegitimidad sexual popular" fue entendida como un problema político y económico. En primer lugar, es conveniente mencionar que para M. Foucault (2008), el surgimiento de la sociedad burguesa y la conformación del Estado Moderno, hizo que la familia apareciera como un espacio dentro de la cual se saturan las sexualidades, se establecen los comportamientos de los individuos; una red de placeres-poderes articulados en puntos múltiples y con relaciones transformables, un mecanismo incitador y articulador de discursos en torno a la sexualidad, que a su vez se encuentra contenido en la distribución de los cuerpos en el espacio (separación entre adultos y niños), segregación entre varones y muchachas, así como también miedos, secretos, métodos de vigilancia sugeridos a los padres, la presencia valorada y a la vez temida de los sirvientes, etc. Se establece una estructura social que contienen en sí misma todas las sexualidades y las reproduce, las contiene y las proyecta hacia el espacio público para afianzar los planes establecidos y aumentar así el poder del Estado sobre los cuerpos (Foucault, 2008).

Desde esta perspectiva y en la línea de los Estudios de Género, al interior de la familia se instauran deberes sociales, morales y sexuales para hombres y mujeres -a partir de su naturaleza biológica- que pongan en ejercicio desde la infancia. Ambos sexos deben controlar sus impulsos y conducirlos hacia la reproducción de nuevas vidas que en el futuro se convertirán en futuras madres, ciudadanos y trabajadores. Así, dentro de la familia, las mujeres se convierten en madres y esposas, mientras que los hombres asumen su responsabilidad como padres y trabajadores. Todo un sistema sexo/género en el que la naturaleza de machos y hembras es transformada, al interior de la familia, en un producto de la cultura. De esta forma, la naturaleza del sexo queda borrada por la cultura y por la producción de "hombres" y "mujeres".

Lo relevante de esta cuestión, es que tal como plantea Gayle Rubin (1986), el parentesco y el matrimonio siempre forman parte de sistemas sociales totales, y siempre están ligados con ordenamientos económicos y políticos. Al interior de la familia se produce un disciplinamiento de las conductas sociales y sexuales, se prohíbe el incesto, se establece como norma la heterosexualidad y se desarrolla la división asimétrica de los sexos. A raíz de ello, los sexos son divididos y deformados, y los niños andróginos y bisexuales son transformados en niños y niñas. De esta forma, la conscripción del individuo en los sistemas de parentesco, que han sido producidos política y económicamente, deja huellas en su psique, por cuanto ha determinado -a partir de la sexualidad biológica-, lo que es propio de las mujeres y de los hombres. 
Así pues, la familia legalizada por el matrimonio, articularía dos espacios: uno privado/familiar/femenino y otro público/político/masculino. (Pateman 1995), En ellos mujeres y hombres asumirían sus deberes y funciones sociales para el funcionamiento del Estado y la economía capitalista. En términos concretos, garantizarían la producción y reproducción de la mano de obra en un contexto social y económico ideal.

En segundo lugar y en función de lo expuesto, es relevante señalar que coincidentemente en Chile, el proyecto "integracionista" de los médicos y las "visitadoras sociales", tuvo como objetivo central iniciar un proceso modernizador del Estado y de la economía capitalista. Sin duda alguna, el control de la crisis y la recuperación del Estado, no podrían hacerse efectivas, sino era a partir de una praxis política que contemplara hasta los espacios más recónditos de la vida del cuerpo del pueblo (Illanes 2006: 15). Nuevamente, el restablecimiento del orden social, político y económico, sería posible por medio de toda una red de discursos y saberes científicos dispuestos a reorganizar las políticas del Estado y al individuo mismo.

Según María Angélica Illanes (2006), en este contexto y desde el punto de vista ideológico-político, la “ilegitimidad” atentaba contra la familia y tenía graves consecuencias en el crecimiento de la población y la vitalidad de la raza. Como consecuencia de estos principios, se estableció científicamente, que la constitución legal de las familias populares, incitaría la conformación de un nuevo orden social y sexual destinado a dirigir y encausar los impulsos sexuales de los individuos hacia la reproducción de la vida y de la fuerza de trabajo al interior del matrimonio, haciendo prevalecer un nuevo orden social, político y económico funcional al proyecto de las elites. Hombres y mujeres, asumirían roles sociales y sexuales de padre y madre; y como resultado asumirían como propia la tarea de salvaguardar las bases del Estado y de la economía nacional. De modo contrario, los amores ilegítimos, continuarían agravando la crisis del pueblo y promovería la desarticulación de todo el sistema.

Como resultado de lo expuesto, consideramos que la familia, aparece en el discurso médico y asistencial como una ficción bien fundada (Bourdieu 1997:128). A través de diversas estrategias, médicos y "visitadoras" buscaron legitimarla en los cuerpos dañados del pueblo, mediante la institución de unos valores y sentimientos adecuados para garantizar la integración que es la condición de la existencia y de la persistencia de esta unidad. Una labor de institución que según Bourdieu (1997), busca incidir en el 
control y elaboración de un objeto afectivo y socialización de la libido. Solo así la familia podría perpetuarse y contribuir en que sus integrantes velaran por la satisfacción de todos sus intereses y por consecuencia, dar solución a los problemas médicos y sociales que tanto les afectaban.

Dicho todo esto entonces, la familia legal, monoparental y heterosexual, cobró vital importancia para los médicos y las "visitadoras sociales", porque a su parecer dirigiría los impulsos sexuales ilegítimos del pueblo hacia la reproducción; pero además porque lograría instituir en la cultura una serie de deberes sociales, morales y sexuales en los cuerpos de los hombres y las mujeres. Desde esta perspectiva, la satisfacción de los impulsos sexuales al interior de la familia promovería la responsabilidad social de los sujetos y a la vez evitaría la propagación de las enfermedades venéreas, el abandono de menores, la prostitución, los vicios, etc. Según ellos/as, esta situación calmaría las iras provocadas por la pobreza, la muerte y la enfermedad del pueblo y dirigiría la satisfacción de las demandas sociales hacia el interior del hogar familiar.

En síntesis, la familia aparece como una la ideología política y sexual que sirve como matriz de representación de las conductas sexuales del pueblo y a partir de la cual es posible comprender la ilegitimidad como un problema político y económico. En este sentido y en la medida en que la familia fue considerada la célula orgánica de la sociedad y del progreso; cualquier conducta sexual que se expresara fuera de sus marcos, sería considerada ilegal e ilegítima. Así pues, los médicos y las visitadoras sociales, a partir de su ideología, fueron quienes definieron en sus artículos las conductas sexuales aceptadas como verdaderas. En suma, elaboraron una "política general de la verdad" (Foucault, 1992), destinada hacia el poder político institucional y hacia la economía capitalista.

\section{Conclusiones.}

A razón de todos los antecedentes expuestos en estas páginas, podemos concluir en primer lugar que el escenario histórico de crisis social, económica y política comprendido entre 1927 y 1937, así como la fundación del Estado Asistencial Sanitario (1925), promovieron el interés de los médicos y las visitadoras sociales para desarrollar investigaciones científicas que les permitieran identificar las causas de los problemas médicos y sociales del pueblo, para diseñar políticas públicas efectivas para calmar el descontento social e incorporar al pueblo al nuevo Estado. 
A partir de este ejercicio científico e intelectual, reconocieron que las prácticas sexuales libres y espontáneas del pueblo eran uno de los factores que incidía directamente en la proliferación de los males de la época: las enfermedades venéreas, los vicios, la mortalidad infantil, la miseria social, etc. Como resultado de ello, abordaron la "ilegitimidad" como un problema moral que, a su juicio, tenía graves repercusiones sobre la vida y la salud del pueblo. Por ello consideraron que resultaba necesario llevar a cabo una política sexual, que desde el Estado fuese capaz de legalizar las uniones libres entre hombres y mujeres para fundar una sociedad que avanzara en conjunto hacia el bienestar de toda la colectividad y asegurara el progreso económico de la nación.

Es así como la familia monoparental heterosexual apareció en el discurso médico y asistencial como la institución que podría, por una parte, disciplinar los impulsos sexuales del pueblo; y por otra, asegurar el progreso de la nación. De esta manera, la moral/sexual instaurada por los médicos y las visitadoras sociales se legitima, políticamente, como verdadera, en la medida en que es producida y articulada desde el Estado y la ciencia. En consecuencia, de ello, los/as profesionales pusieron en marcha una política general de la verdad (Foucault, 2009:198), que desconoció, marginó y silenció cualquier otra práctica sexual que no se llevara a cabo al interior de la familia monoparental y heterosexual.

Por consiguiente, médicos y visitadoras establecieron las normas de comportamiento social y sexual ideales, vinculadas a categorías de género, que eran funcionales a su propio proyecto político y económico. En este sentido, por ejemplo, establecieron que todo acto sexual debía llevarse a cabo al interior de la unión matrimonial, que las mujeres debían asumir su función social maternal como el fin de su existencia y los hombres debían controlar sus impulsos sexuales y asumir su función social paterna. Lo significativo de ello, es que la familia monoparental heterosexual sería funcional al Estado y a la economía capitalista, y por ello, todos los roles sociales y sexuales producidos y reproducidos en ella, reafirmarían el proyecto político y económico de quienes los sustentaron política e ideológicamente.

La familia no solo operó en el discurso médico y asistencial como un modelo ejemplar de las conductas sexuales, sino que además fue considerada como la base de toda sociedad bien organizada, política y económicamente. Por este motivo, podemos concluir que la ilegitimidad sexual popular fue considerada un problema político y económico, en la medida que impedía la conformación de hogares familiares y 
promovía la proliferación de los males médicos y sociales que agudizaban la crisis política y económica, y amenazaban la desestructuración de todo el cuerpo social.

\section{Referencias bibliográficas}

Allende S. (1939). La realidad médico-social chilena, Santiago, Chile: Ministerio de Salubridad.

Bourdieu P. (1997). Razones Prácticas. Sobre la Teoría de la Acción. Barcelona: Anagrama.

Canturial R. y Rodríguez A. (1936). "Algunas ideas sobre la forma en que debe abordarse el problema de las enfermedades venéreas.” Revista de Asistencia Social. V. (1): 21-35.

Carrasco R. (1928). «Reflexiones de una iniciada en el Servicio Social.» Servicio Social, II. (1): 10-14.

Córdova L. (1932). «Primer Código Sanitario de Chile.» Servicio Social. VI. (4) : 264-286.

De Barbieri T. (1993). «Sobre la categoría de Género: Una introducción teórico metodológica.» Debates en Sociología: 145-169.

De Bray L. (1933). «Dlincuencia juvenil y servicio social.» Servicio Social, VII. (3): 147-156.

Foucault M. (2008). Historia de la Sexualidad. Vol. I. La voluntad de saber. Buenos Aires: Siglo veintiuno editores. (2009). La arqueología del Saber. Madrid: Siglo veintiuno editores.

Gajardo S. (1929). "Las deficiencias del hogar como factor de delincuencia de menores.” Servicio Social, III. (4): 199-205.

Illanes M. (1993). En el nombre del pueblo, del Estado y de la ciencia, (...) Historia social de la salud pública, Chile 1880/1973 (hacia una historia social del siglo XX). Santiago: Colectivo de Atención Primaria.

(2003) "Las Escribas. Seducción sexual popular y Estado nacional. Chile, 1928-1948". Proyecto Fondecyt, N 1020135: Santiago. (2006). Cuerpo y sangre de la política. La construcción histórica de las "visitadoras sociales" 1887-1940. Santiago: LOM.

Mac Auliffe A. (1932). «La conformación del hogar del pueblo chileno.» Servicio Social. VI (4) : 287304.

Mulle M. (1930) “La educación moral y social del niño Servicio Social. VI. (1): 25-33.

Pateman C. (1995). El contrato sexual. Itzapalapa: Ediciones Anthoropos. 
Recchione H. (1935). «Orientaciones sobre el porblema médico legal del niño.» Revista de Asistencia Social. IV (4): 574-590.

Reich W. (1985). La Revolución sexual. Para una estructura de carácter autónoma del hombre. México: Editorial Artemisa.

Rioseco E. (1934). «Profilaxis de las enfermedades venéreas.» Revista de Asistencia Social, III. (3): 320331.

Rubin G. (1986). «El tráfico de mujeres: notas sobre la economía política del sexo.» Revista Nueva Antropología. VIII, (30): 95-145.

Salazar Gabriel y Pinto Julio. (2002). Historia Contemporánea de Chile. La economía: mercados, empresarios y trabajadores. Tomo III. Santiago: LOM.

Valdés I. (1928). «El huérfano.» Servicio Social. II. (1): 190-199. 